\title{
Secuencias de Pregunta, Respuesta y Seguimiento en Situaciones de Juego y Cuentos en el Nivel Inicial
}

\section{Initiation, Response, and Follow-Up Sequences in Play Situations and Story Reading in Preschool}

\author{
Mariana Sartori ${ }^{1,2}$, Carolyn Ortiz ${ }^{3}$, Paulina Pizarro ${ }^{4}$, Daniela Jauck ${ }^{1,2}$, Alejandra Stein ${ }^{5,6}$, \\ Florencia Alam ${ }^{5}$, Celia Rosemberg ${ }^{5,6}$, Olga Peralta ${ }^{1,2}$ y Katherine Strasser ${ }^{7}$ \\ ${ }^{1}$ Instituto Rosario de Investigaciones en Ciencias de la Educación \\ ${ }^{2}$ Consejo Nacional de Investigaciones Científicas y Técnicas, Universidad Nacional de Rosario \\ ${ }^{3}$ Escuela de Fonoaudiología, Universidad Mayor \\ ${ }^{4}$ Pedagogía en Educación Parvularia, Universidad Diego Portales \\ ${ }^{5}$ Centro Interdisciplinario de Investigaciones en Psicología Matemática y Experimental \\ ${ }^{6}$ Universidad de Buenos Aires \\ ${ }^{7}$ Escuela de Psicología, Pontificia Universidad Católica de Chile
}

\begin{abstract}
El lenguaje que utilizan las maestras tiene importantes asociaciones con el lenguaje de los niños/as a su cuidado. Un aspecto importante es el tipo de preguntas que realizan las maestras y la forma en que dan seguimiento a las respuestas de los niños/as, lo que se conoce como secuencias pregunta-respuesta-seguimiento (PRS). En el nivel inicial, estas secuencias han sido estudiadas mayormente en situaciones de lectura de libros. En la investigación que aquí se presenta se comparan secuencias PRS en cuento y juego. Participaron 10 jardines de infantes de la provincia de Entre Ríos, Argentina, seleccionados mediante un muestreo intencional. Específicamente, participaron 10 maestras y 300 niños/as. Desde un enfoque microanalítico, se analizaron 13 grabaciones de audio en situaciones de lectura de cuentos y 16 durante juegos de rincón. Para evaluar las diferencias en el tipo de preguntas, respuestas y seguimiento en ambas situaciones se utilizó la prueba $\chi^{2}$. Además, se realizó un análisis secuencial de la relación entre el tipo y complejidad de la pregunta, de la respuesta y del seguimiento. Los resultados muestran que en ambas situaciones predomina el uso de preguntas de baja complejidad o literales y de seguimientos de bajo nivel, tales como confirmación, evaluación, falsificación y respuesta. Se destaca que el tipo y la complejidad de la pregunta que realiza la maestra determina fuertemente la complejidad de la respuesta de los niños/as. Se discuten las implicancias didácticas del presente estudio, específicamente en cuanto a la práctica y formación docente.
\end{abstract}

Palabras clave: lenguaje, secuencias PRS, nivel inicial, cuento, juego

\begin{abstract}
The language used by teachers has been found to be closely associated with the oral language of the children in their care. The types of questions that teachers ask and the way they follow-up children's responses constitute initiationresponse-follow-up (IRF) sequences, which are an important aspect of teacher-student verbal exchanges. In preschool, these sequences have been studied mostly in book reading interactions. The present study compares IRF sequences in 2 situations: book reading and play. The sample comprised 10 preschools, purposively selected in the province of Entre Ríos, Argentina. Specifically, the participants were 10 teachers and 300 children. Employing a micro analytical approach, 13 audio recordings of book reading and 16 of corner play were analyzed. To evaluate the differences between the types of questions, responses, and follow-up in the 2 situations, the $\chi^{2}$ test was used. In addition, a sequential analysis was performed of the relationship between the type and complexity of the question, of the response, and of the follow-up. Results show that both situations predominantly feature low complexity or literal questions and low-level follow-ups, such as confirmation, evaluation, falsification, and response. Relevantly, the type and complexity of the question asked by the teacher strongly determine the complexity of the children's response. The didactic implications of this study are discussed, specifically regarding teaching practices and teacher education.
\end{abstract}

Keywords: language, IRF sequences, preschool, story, play

Mariana Sartori iD https://orcid.org/0000-0001-6921-5462

Esta investigación forma parte del Proyecto REDES-CONICYT (Chile), "Lexicon, narration and argumentation: Cognitive development, ecological challenges and educational proposals". Instituciones participantes: Universidad Diego Portales, Pontificia Universidad Católica de Chile, Centro Interdisciplinario de Investigaciones en Psicología Matemática y Experimental e Instituto Rosario de Investigaciones en Ciencias de la Educación, Argentina.

La correspondencia relativa a este artículo debe ser dirigida a Mariana Sartori, Instituto Rosario de Investigaciones en Ciencias de la Educación, 27 de febrero 210 bis, Rosario, Santa Fe, Argentina. Email: sartori@irice-conicet.gov.ar 
El lenguaje oral desarrollado durante la etapa preescolar es esencial para el aprendizaje de la lectura y escritura y, por lo tanto, de las diversas disciplinas en la escuela primaria (Cain \& Oakhill, 1999; Cain et al., 2004). En relación con este tema, diversas investigaciones en el marco de la lingüística sistémico funcional (LSF) mostraron que el lenguaje utilizado por las maestras varía dependiendo de la actividad que se esté realizando (e.g., Ibañez et al., 2018; Strasser et al., 2018; Treviño et al., 2015).

En esta línea, numerosos estudios analizaron las preguntas efectuadas por las maestras en situaciones de lectura de libros infantiles y durante la discusión de esas lecturas (e.g., Dickinson \& Smith, 1994; Mascareño et al., 2017; Reese et al., 2003). Si bien existen otros momentos en el preescolar, tales como el juego de rincón, la mayoría de las investigaciones se centró en la lectura de cuentos. La mayoría de los estudios que indagaron otras situaciones de la jornada escolar informó resultados de países de altos ingresos (e.g., Gest et al., 2006; Meacham et al., 2014). Esto representa un problema, porque existen condiciones culturales y estructurales que podrían incidir en los resultados, tales como cantidad de niños/as por adulto, acceso a materiales para el juego de ficción y formación del personal de sala. Como muchos otros países latinoamericanos, Argentina, donde se llevó a cabo el presente estudio, se caracteriza por una fuerte fragmentación socioeconómica, que se expresa en una importante variación en las dimensiones de vivienda, ocupación y cantidad y calidad de la educación formal, que determina condiciones de vida y desarrollo marcadamente diferentes entre los niños/as.

En este marco, los objetivos del presente estudio fueron analizar las interacciones comunicativas entre maestras y niños/as en situaciones de lectura de cuentos y de juegos de rincón y comparar ambas situaciones en una muestra conformada por maestras y niños/as de 5 años, de nivel socioeconómico (NSE) bajo y medio bajo.

\section{Interacciones Pregunta-Respuesta-Seguimiento (PRS)}

Las preguntas de las maestras han sido estudiadas tradicionalmente en el contexto de secuencias pedagógicas que consideran inicio, respuesta y seguimiento (PRS, proveniente del inglés initiation, response, follow-up, IRF; Mehan, 1979; Preiss, 2009; Sinclair \& Coulthard, 1975). Cada secuencia se inicia con una pregunta formulada por la maestra, seguida por una respuesta del niño/a y cerrada por un seguimiento de la maestra. La estructura de la PRS desempeña dos funciones en el aula: por un lado, permite al docente manejar la interacción en la sala de clases y, por otro, lo sitúa en el rol de quien maneja un mayor conocimiento en la interacción.

La estructura PRS remite al concepto de sistema de ayuda para la adquisición del lenguaje (SAAL, del inglés language acquisition support system, LASS; Bruner, 1986). El SAAL está constituido por rutinas o formatos que los adultos crean con los niños/as. Estos formatos consisten en interacciones contingentes y reversibles: la respuesta de cada miembro depende de la contestación previa del otro.

Los investigadores que estudiaron las interacciones PRS entre maestras y niños/as que asisten al preescolar postularon, en general, que dichas interacciones deben ser cognitivamente desafiantes, ayudar al aprendizaje y ser el andamiaje para la construcción del conocimiento (Carlsen, 1991; Chafy \& Elkhouzai, 2014; Wang \& Wang, 2013). Esto implica que es importante tanto el análisis de la pregunta, caracterizada según su complejidad y tipo, como el análisis del seguimiento, caracterizado según su bajo o alto nivel cognitivo (Mascareño et al., 2017).

La complejidad de la pregunta se refiere a la distinción entre preguntas literales, cuya respuesta está disponible a través de la percepción o la memoria, y preguntas inferenciales, en las cuales la información no está disponible en el contexto inmediato o no ha sido previamente proporcionada (Mascareño et al., 2017; Sembiante et al., 2018; van Kleeck et al., 2006). Las preguntas inferenciales requieren información externa relevante para la elaboración de inferencias o conclusiones, por lo que propician discusiones con lenguaje abstracto y explicaciones de causa-efecto (Sembiante et al., 2018; van Kleeck et al., 2006).

Las preguntas también pueden ser clasificadas según su nivel de restricción, es decir, pueden ser cerradas o abiertas (Tornero et al., 2015). Las preguntas cerradas están destinadas a evaluar si los conocimientos de los niños/as son correctos, mientras que las preguntas abiertas permiten que los niños/as elaboren sus ideas, al ampliar el abanico de respuestas posibles. Las preguntas abiertas propician un nivel de pensamiento más alto, al aumentar el compromiso cognitivo (Smart \& Marshall, 2013), propiciando la aparición de respuestas más extensas (Ramírez-Esparza et al., 2014; Zimmerman et al., 2009) y conversaciones cognitivamente más desafiantes (Mascareño et al., 2017). 
Los seguimientos que realizan las maestras a las respuestas de los niños/as son muy relevantes, porque dan señales de interés, proporcionando información y respuestas en función de las intervenciones de los niños/as, lo que favorece el aprendizaje de nuevos conocimientos (Carlsen, 1991; Chin, 2006; Wang \& Wang, 2013). Los seguimientos de bajo nivel o de menor complejidad son muy comunes en las aulas de nivel inicial (Dickinson et al., 2003; Mascareño et al., 2016). Los seguimientos de alto nivel estimulan el vocabulario de los niños/as (Wasik \& Bond, 2001), porque extienden la participación infantil en la conversación de la clase (Mascareño et al., 2016) y permiten el desarrollo de un pensamiento más elaborado (Chin, 2006). Estos seguimientos se expresan mediante expansiones de la respuesta infantil (Mascareño et al., 2016), sugerencias, preguntas (Chin, 2006) o reelaboraciones de las contribuciones de los niños/as (Chafy \& Elkhouzai, 2014). Los seguimientos más complejos posibilitan un mayor uso de contenido inferencial y de palabras sofisticadas, es decir, poco frecuentes en el lenguaje oral, pero que se encuentran en textos escritos. Estas palabras difícilmente se aprenden en la cotidianeidad, al no estar presentes en la experiencia espontánea, y requieren, por lo tanto, de una enseñanza explícita (Beck et al., 2013).

\section{Interacciones PRS en Situaciones de Lectura de Cuentos y Juegos de Rincón}

En relación con las interacciones PRS en situaciones de lectura de cuentos, diversos estudios muestran, tanto en el nivel inicial como en la escuela primaria, que existe un predominio de preguntas, respuestas y seguimientos de bajo nivel (e.g., Chafy \& Elkhouzai, 2014; González et al., 2008; Hiebert et al., 2003; Preiss, 2009). Por ejemplo, Tornero et al. (2015) encontraron que, aunque las preguntas realizadas por las maestras de preescolar en torno a la lectura de cuentos eran con mayor frecuencia abiertas, estas eran de baja complejidad cognitiva, puesto que la mayoría era literal. Así, las preguntas de las maestras son en su mayoría literales y los seguimientos son evaluaciones destinadas a verificar si las respuestas son correctas o incorrectas, siendo escasos los seguimientos elaborados (Mascareño et al., 2016; Ortiz \& Lissi, 2019; Preiss, 2009).

Por otro lado, diversos estudios analizaron determinadas características del habla utilizada por las maestras en situaciones de juego de rincón. El juego de rincón consiste en establecer áreas de trabajo en distintos sectores de la sala en los que generalmente los niños/as eligen dónde desean jugar. Los rincones pueden ser de construcción, con juegos y materiales, como bloques, y de dramatización, con peluches y muñecas, entre otros. Kontos (1999) analizó el habla y tipo de actividad durante situaciones de juego en salas incluidas dentro del programa Head Start de Estados Unidos. Su estudio encontró que aproximadamente el $75 \%$ de las emisiones docentes estaba orientada a promover o apoyar el desarrollo del juego mediante afirmaciones o preguntas, a proporcionar asistencia práctica (facilitar el acceso a materiales) y retroalimentar positivamente el desempeño del niño/a y solo el $5 \%$ se orientaba a regular el comportamiento infantil (leer, jugar con ellos, estimular las relaciones entre pares).

Una serie de trabajos identificó relaciones entre el habla de las maestras en el transcurso de las situaciones de juego y la participación de los niños/as y el desarrollo del lenguaje infantil (Dickinson \& Porche, 2011; Migdalek \& Rosemberg, 2014; Rosemberg, 2008; Sarlé, 2000). Por ejemplo, Manrique y Rosemberg (2009) analizaron interacciones entre maestras y niños/as de 4 y 5 años de edad en salas de preescolar, concluyendo que, a nivel global, la proporción de participación de niños/as y maestras era similar. Sin embargo, esta proporción varió en fases específicas del juego: las maestras produjeron más turnos al inicio del juego, al tener que organizarlo y explicarlo, y los niños/as produjeron mayor cantidad de turnos cuando eligieron el tipo de juego en el que iban a participar. Por otra parte, en relación al impacto del habla docente en el desarrollo del lenguaje infantil, Dickinson y Porche (2011) encontraron que la utilización de vocabulario sofisticado por parte de las maestras en situaciones de juego se vinculó con la comprensión lectora que los niños/as mostraron en cuarto año de educación primaria.

En cuanto al seguimiento de los adultos frente a intervenciones infantiles, Rosemberg (2000) analizó las interacciones de niños/as preescolares y de primer grado de educación primaria con sus madres y maestras. Se observó que las intervenciones de estas contribuyeron a aumentar la complejidad y extensión temporal del juego. Además, la sincronización de las intervenciones entre los participantes, la atención compartida frente a un tema y las estrategias proporcionadas por los adultos (reestructuraciones y expansiones) facilitaron la participación infantil. Estas reestructuraciones y reconceptualizaciones de las emisiones ampliaron los conocimientos acerca de objetos y eventos, toda vez que permitieron a los niños/as relacionar sus conocimientos previos con aquellos que debían aprender.

Otros estudios compararon la interacción de maestras y niños/as en diferentes situaciones del aula. Por ejemplo, Girolametto et al. (2000) compararon el lenguaje de las maestras durante situaciones de lectura y 
juego y encontraron que, en ambos casos, la cantidad de preguntas que llamaron de control de la conversación se correlacionaba directamente con la cantidad de turnos que tomaban los niños/as. También observaron que estas preguntas eran más frecuente durante el juego, mientras que las preguntas cerradas y de control de respuestas eran más frecuentes en la lectura de cuentos. Adicionalmente, los autores extendieron estos resultados a los seguimientos (Girolametto \& Weitzman, 2002), encontrando que también era más frecuente en situaciones de juego y que existía una correlación directa con el lenguaje de los niños/as.

Gest et al. (2006), por su parte, estudiaron el habla dirigida a los niños/as durante juego libre, comidas y lectura de cuentos en 20 salas del programa estadounidense Head Start. En su estudio, no encontraron diferencias en la cantidad de habla dirigida al niño/a, pero sí en sus características. Las maestras exhibieron mayor habla descontextualizada durante las comidas y mayor habla sofisticada durante la lectura de cuentos. Por otro lado, el habla "de ficción" solo se observó durante el juego libre. Esto no es sorprendente, ya que el juego libre representa una excelente oportunidad para que las maestras se involucren y, sin cooptar el juego, medien diferentes aspectos de lenguaje, conocimientos o, incluso, relaciones interpersonales. De hecho, otro estudio (Meacham et al., 2014) examinó las preguntas de las maestras y las respuestas de los niños/as durante el juego de ficción y encontró que, aunque el habla de las maestras no era particularmente sofisticada (predominaban las preguntas cerradas), estaba fuertemente relacionada con la situación de ficción del juego. Las investigadoras argumentaron que el predominio de preguntas cerradas durante el juego de ficción podría deberse a que, en los contextos de la vida real representados por los niños/as, predomina este tipo de interacciones (e.g., ¿En qué talla lo quiere? ¿Quiere leche en su café?). Así, Gest et al. (2006) plantean que la estimulación del lenguaje en el juego sociodramático debiera evaluarse no solo en cuanto al desarrollo de aspectos como la sintaxis y el vocabulario, sino también de la pragmática.

En otro estudio (Strasser et al., 2018) se comparó el lenguaje de maestras y niños/as chilenos en ocho tipos de situaciones, incluidos juego libre y lectura. Se encontraron diferencias en casi todas las variables de lenguaje, pero no se comparó específicamente juego con lectura. Strasser et al. (2018) también han mostrado que en Chile, aunque se lo considera importante y recomendable, el juego de ficción y guiado es poco frecuente. Cabe destacar que en Argentina, el juego de ficción en rincones guiado por la maestra es una actividad muy usual (Sarlé, 2000, 2006).

En síntesis, las investigaciones previas que analizaron las interacciones PRS en situaciones de lectura de cuento pusieron de manifiesto que en ellas predominan las preguntas y los seguimientos de bajo nivel (Chafy \& Elkhouzai, 2014; González et al., 2008; Hiebert et al., 2003; Mascareño et al., 2016; Ortiz \& Lissi, 2019; Preiss, 2009; Tornero et al., 2015). Por su parte, aquellos estudios centrados en situaciones de juego atendieron a determinadas características del habla docente, tales como la sofisticación del vocabulario, la cantidad de turnos y la función pragmática, así como a las relaciones entre las características mencionadas y la participación de los niños/as en las situaciones y el desarrollo del lenguaje infantil (Dickinson \& Porche, 2011; Kontos, 1999; Manrique \& Rosemberg, 2009; Rosemberg, 2008; Sarlé, 2000). No obstante, en ninguno de los trabajos mencionados se analizó las preguntas efectuadas por las maestras ni su impacto en las respuestas de los niños/as. Los escasos trabajos que analizaron comparativamente el discurso docente en ambos tipos de situaciones identificaron diferencias en los tipos de preguntas formuladas por las maestras (Gest et al., 2006; Girolametto et al., 2000; Meacham et al., 2014). Sin embargo, no se encontraron estudios indagando las secuencias PRS en situaciones de juego de rincón y analizando el impacto de las preguntas de las maestras en las respuestas infantiles y en los seguimientos. Tampoco se hallaron estudios que comparaban estas secuencias en situaciones de juego y de lectura de cuentos. Por tanto, el presente estudio constituye un novedoso aporte en esa dirección.

\section{El Presente Estudio}

De los resultados de las investigaciones revisadas, se desprende que el habla de las maestras en nivel inicial durante la lectura de cuentos no necesariamente es extrapolable a situaciones de juego. Dada la naturaleza de las situaciones, probablemente algunas formas de secuencias PRS sean más adecuadas en una situación que en otra. Por ejemplo, si bien las preguntas abiertas e inferenciales referidas a un cuento que se acaba de leer tienen un impacto positivo en el lenguaje de los niños/as (Ramírez-Esparza et al., 2014; van Kleeck et al., 2006; Zimmerman et al., 2009), no queda claro qué rol podrían cumplir durante el juego.

El objetivo de la presente investigación fue estudiar y comparar las secuencias PRS en situaciones de juego y cuento en salas de preescolar y determinar la asociación entre los tipos de preguntas de las maestras, los tipos de respuestas de los niños/as y los niveles de seguimientos de las maestras. Específicamente, el 
propósito fue identificar similitudes y diferencias entre la frecuencia de las preguntas, las respuestas y los seguimientos en las situaciones de juego y cuento.

\section{Contexto del Estudio}

En esta investigación se analizaron interacciones dentro del aula entre maestras y niños/as de 5 años de edad, registradas en el marco de un proyecto de intervención llevado a cabo por el Consejo Nacional de Investigaciones Científicas y Técnicas (CONICET), la Fundación Arcor y el Consejo General de Educación de la Provincia de Entre Ríos, Argentina: "Proyecto de promoción del desarrollo lingüístico y cognitivo de niños pequeños" (Rosemberg \& Borzone, 2010). Los fondos para realizar este proyecto se obtuvieron del "Programa de Promoción del Desarrollo Lingüístico y Cognitivo para los Jardines de Infantes de la Provincia de Entre Ríos" dirigido por la séptima autora de este artículo y Ana María Borzone.

Durante el proyecto, las maestras participaron de cuatro jornadas intensivas de formación específica acerca de la importancia de la interacción para el desarrollo del lenguaje y la alfabetización temprana, así como de la implementación didáctica de las situaciones que configuran la estructura típica en el preescolar (Rosemberg et al., 2010). Las capacitaciones estuvieron a cargo de los equipos técnicos de los ministerios y de los directivos que habían participado previamente de ocho jornadas de formación docente. Asimismo, como parte del programa, había orientadoras educacionales, quienes eran maestras en comisión de servicio que habían recibido formación especial e intensiva. Las orientadoras acudieron a las aulas con una frecuencia quincenal y realizaron grabaciones de audio de jornadas escolares completas durante el año 2011. La estructura típica de la jornada escolar de los preescolares incluidos en el presente estudio comprende situaciones de intercambio en la ronda, lectura de cuentos, juego, escritura de palabras y textos, así como alimentación y aseo (Lineamientos curriculares para la educación inicial, 2008).

\section{Método}

\section{Participantes}

Los datos que se analizaron en este estudio corresponden a niños/as y maestras de 10 jardines de infantes estatales urbanos de la provincia de Entre Ríos, Argentina. En el presente estudio participaron 10 maestras y 300 niños/as, seleccionados mediante un muestreo intencional (Marradi et al., 2007). Los niños/as provenían de hogares de NSE bajo y medio bajo. El $72,10 \%$ de las madres tenía escolaridad primaria (7 años de escolaridad formal), el 26,5\%, escolaridad secundaria completa o incompleta (8 a 12 años de escolaridad) y el $1,30 \%$, educación universitaria.

\section{Materiales}

Las diferentes situaciones de enseñanza que forman parte del corpus de la quinta y séptima autora (Rosemberg et al., 2010) tuvieron lugar a lo largo de las jornadas registradas. Para este trabajo se seleccionaron y tomaron como objeto de estudio la totalidad de los registros de las situaciones de lectura de cuentos y juego en rincones. El corpus que se analiza comprende 13 lecturas de cuentos y 16 juegos en rincones llevadas a cabo por un total de 10 docentes. Las mismas maestras fueron grabadas mediante audio y video en ambos tipos de situaciones.

En relación a los materiales empleados, los mismos fueron especialmente seleccionados como parte del programa. Se emplearon libros de cuentos y de textos expositivos y diversos juguetes que permitían juegos reglados y dramáticos.

Las situaciones de juego se organizaban en tres fases. En la conversación inicial la maestra y los niños/as planificaban la situación de juego, conversaban sobre los objetos disponibles en cada rincón de la sala, típicamente un sector con juguetes para jugar a "la casita", un sector con rompecabezas y juegos de mesa, otro con libros infantiles y útiles para dibujar y pintar y uno de construcciones con bloques, autos y camiones de juguete. Los niños/as seleccionaban el rincón al que iban a integrarse y lo que iban a hacer allí. El segundo momento consistía en el juego propiamente dicho. La situación terminaba con un intercambio en el que los niños/as relataban a qué habían jugado y comparaban lo que habían planificado hacer con aquello con lo que efectivamente habían jugado. 
Las situaciones de lectura de cuentos tenían frecuentemente una estructura compuesta por tres partes: la activación de las experiencias y los conocimientos previos de los niños/as en relación con el tema del cuento, la lectura en voz alta por parte de la maestra y, finalmente, la reconstrucción grupal del cuento por parte de los niños/as y de la maestra.

Las grabaciones de las interacciones fueron transcriptas a protocolos para su posterior codificación y análisis.

\section{Procedimiento}

Esta investigación cumplió con los lineamientos para el comportamiento ético en las ciencias sociales y humanidades establecidos por CONICET (Lineamientos para el comportamiento ético, 2006). Se contó con el consentimiento informado por escrito y firmado de los directivos, docentes y familiares a cargo de los niños/as. Los consentimientos fueron tramitados por el Consejo General de Educación de la provincia de Entre Ríos al que pertenecen los preescolares que participaron.

\section{Análisis de Datos}

\section{Sistema de Codificación}

Las transcripciones fueron fragmentadas y la unidad de análisis fue el enunciado, es decir, una emisión de habla de la maestra o del niño/a consistente en una oración independiente con sus oraciones subordinadas, si las hubiere.

El total de enunciados en las 29 interacciones (16 correspondientes a juego de rincón y 13 a lectura de cuento) fue de 9039 (6172 para juego y 2867 para cuento). De estos enunciados, se excluyeron del análisis las emisiones de la maestra que no eran preguntas ni seguimientos, así como las emisiones infantiles que no eran respuestas a una pregunta de la maestra. El total de enunciados analizados fue, por lo tanto, de 5430 (3777 de juego de rincón y 1653 de lectura de cuento).

Cada enunciado fue codificado de acuerdo al tipo de pregunta, respuesta o seguimiento, para lo cual se adaptó un sistema de codificación utilizado por Mascareño et al. (2017), el que se puede ver en la Tabla 1.

El siguiente constituye un ejemplo de secuencia codificada según tipo de pregunta, respuesta y seguimiento:

Maestra: ¿qué se necesita para que el barrilete vuele? (CI2)

Niño: viento. (PRI1)

Maestra: el viento ¡Muy bien! (CBE1)

Tres de las coautoras codificaron en forma separada una interacción completa para acordar procedimientos y criterios. Posteriormente, se realizaron tres rondas de codificación por dos codificadoras independientes capacitadas, con 5, 4 y 8 interacciones por ronda. La concordancia intercodificador reportada es la de la tercera ronda, que incluyó los enunciados de cuatro situaciones de lectura de cuento y cuatro de juegos de rincón. Al final de cada ronda, se efectuó un análisis de los desacuerdos y un refinamiento de las reglas de codificación. Finalmente se revisó la codificación. Para calcular el acuerdo, además del coeficiente Kappa de Cohen se analizó el porcentaje de acuerdo entre codificadoras. Cuando se obtuvo un porcentaje de acuerdo superior al 70\%, las codificadoras continuaron codificando de forma independiente. La Tabla 2 presenta los coeficientes Kappa de Cohen para cada una de las dimensiones de codificación.

Cabe aclarar que la discrepancia entre los altos porcentajes de acuerdo y bajos coeficientes Kappa se explica porque la distribución de frecuencias fue muy baja, es decir, hubo códigos que tuvieron una ocurrencia muy baja. Tal como explican Bruckner et al. (2006), un coeficiente Kappa de 0,3 para una variable que tiene una prevalencia real de $30 \%$ corresponde a una precisión de $80 \%$ en los codificadores, lo cual se aplica a la mayoría de los códigos de este estudio, puesto que la mayoría tiene una prevalencia menor al 30\%. De todas formas, para asegurar la validez de los códigos asignados, la tercera autora del estudio revisó todas las codificaciones. 


\section{Tabla 1}

Sistema de Codificación de las Interacciones PRS

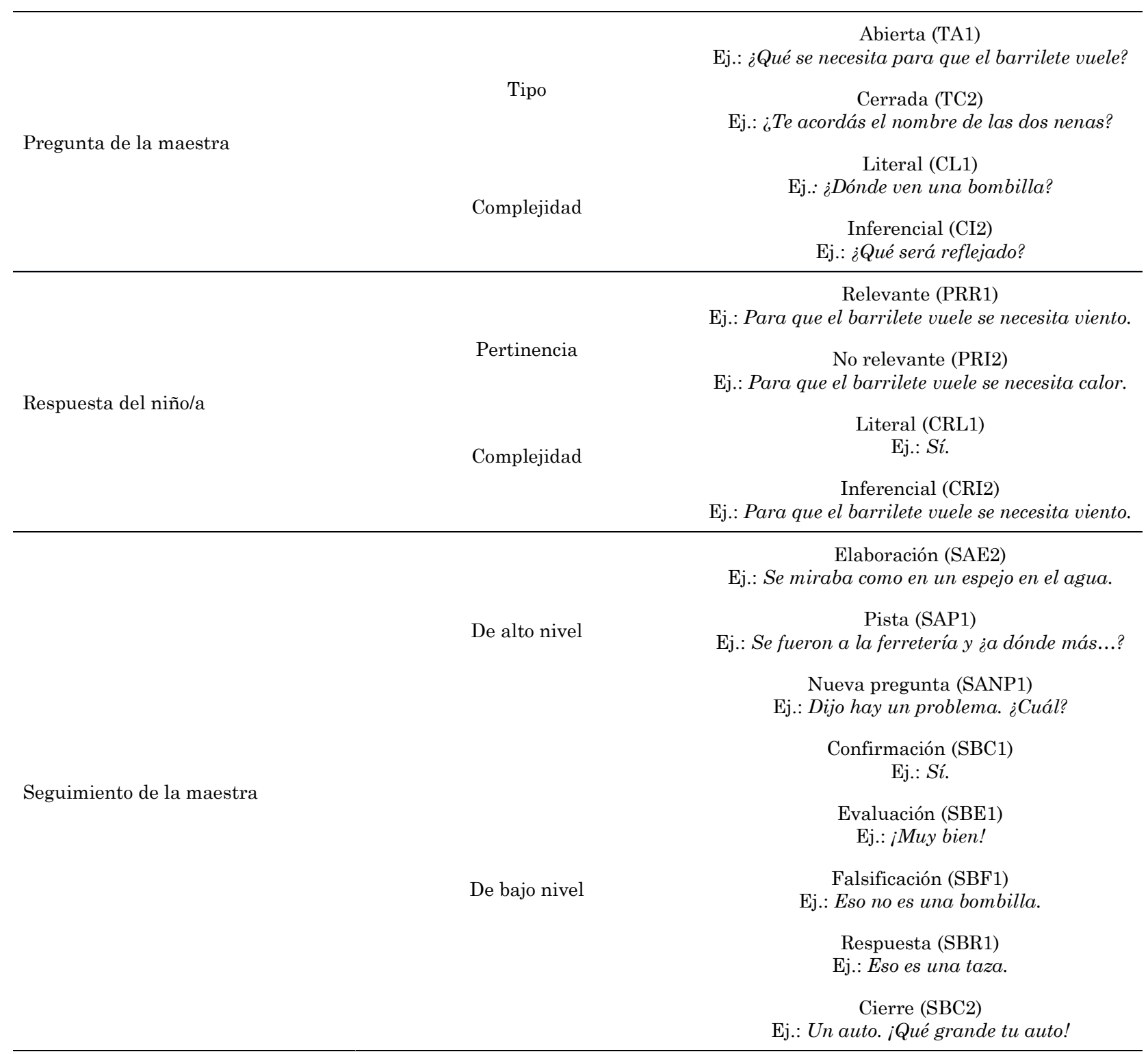

Tabla 2

Promedio de Coeficiente Kappa y Porcentaje de Acuerdo por Dimensión

\begin{tabular}{lccc}
\hline \multicolumn{1}{c}{ Dimensión } & $\begin{array}{c}\text { Promedio } \\
\text { Kappa }\end{array}$ & $\begin{array}{c}\text { Rango de } \\
p\end{array}$ & $\begin{array}{c}\text { Porcentaje de } \\
\text { acuerdo }\end{array}$ \\
\hline Tipo de pregunta o seguimiento & 0,362 & $\begin{array}{c}0,013 \text { a } \\
<0,001\end{array}$ & 76,5 \\
Complejidad de pregunta y respuesta & 0,290 & $\begin{array}{c}\text { Todos } \\
<0,001\end{array}$ & 80,3 \\
Pertinencia de respuesta & 0,320 & $\begin{array}{c}\text { Todos } \\
<0,001\end{array}$ & 97,4 \\
\hline
\end{tabular}




\section{Análisis Cuantitativo}

Se utilizó un enfoque microanalítico, con el que se examinó el conjunto de interacciones PRS. Este tipo de análisis pretende capturar los procesos dinámicos de cambio en las interacciones, con el propósito de observar la novedad psicológica (García Cernaz, 2019). Para evaluar las diferencias entre el tipo de preguntas en las dos situaciones (juego y cuento), se realizaron comparaciones, usando la prueba $\chi^{2}$. Se efectuaron comparaciones entre preguntas abiertas inferenciales y literales en cuento y juego, preguntas cerradas inferenciales y literales en cuento y juego, y otro tipo de preguntas abiertas y cerradas en cuento y juego.

Además, se realizó un análisis secuencial de la relación entre tipo y complejidad de la pregunta, tipo y complejidad de la respuesta, así como el tipo y complejidad del seguimiento, utilizando el programa GSEQ (Software for the Analysis of Interaction Sequences). Este programa permitió calcular la probabilidad de ocurrencia de cada tipo de respuesta para cada tipo de pregunta, así como la probabilidad de ocurrencia de seguimientos de bajo nivel (confirmación, evaluación, falsificación, respuesta y cierre) y de alto nivel (elaboración, pista y nueva pregunta) para cada tipo de respuesta y la probabilidad de ocurrencia de los tipos de seguimientos en función del tipo de pregunta realizada por la maestra. El análisis permitió estudiar de manera separada las situaciones de lectura de cuento y de juego de rincón, así como comparar ambas situaciones para determinar si el tipo de secuencia PRS era similar en ambas o si cada una presentaba particularidades.

\section{Resultados}

\section{Distribución de Interacciones PRS en Situaciones de Juego y Cuento}

Considerando situaciones de juego de rincón y lecturas de cuento, los enunciados emitidos por las maestras fueron 3023, equivalentes al $55,7 \%$ del total (2103 en juego y 920 en cuento), mientras que las emisiones producidas por los niños/as fueron 2407, correspondientes al 44,3\% del total (1674 en juego y 733 en cuento).

En cuanto a las emisiones producidas por las maestras, el 70,8\% fueron preguntas $(72,7 \%$ en juegos y $21,3 \%$, en cuentos) y el $29,2 \%$, seguimientos ( $62,1 \%$ en juegos y $37,8 \%$ en cuentos). En la Tabla 3 se presenta la distribución del tipo y complejidad de las preguntas efectuadas por las maestras.

Tabla 3

Tipo y Complejidad de Preguntas Efectuadas por las Maestras

\begin{tabular}{lcccc}
\hline & \multicolumn{2}{c}{ Juego } & \multicolumn{2}{c}{ Cuento } \\
\cline { 2 - 5 } Tipo y complejidad de pregunta & Frecuencia & $\%$ & Frecuencia & $\%$ \\
\cline { 2 - 5 } Cerrada literal & 898 & 57,8 & 467 & 79,8 \\
Cerrada inferencial & 182 & 11,7 & 73 & 12,5 \\
Abierta literal & 122 & 7,9 & 1 & 0,2 \\
Abierta inferencial & 85 & 5,5 & 30 & 5,1 \\
Cerrada otra & 215 & 13,8 & 14 & 2,4 \\
Abierta otra & 52 & 3,3 & 0 & 0,0 \\
Total & 1554 & 100,0 & 585 & 100,0 \\
\hline
\end{tabular}

En la Tabla 3 se observa que la mayoría de las preguntas efectuadas por las maestras fueron cerradas y literales, más frecuente en situaciones de lectura de cuento que en juego de rincón. En los juegos hubo preguntas abiertas literales y otro tipo de preguntas, como organizativas, de control o pseudopreguntas (por 
ejemplo, "¿Quién más quiere jugar?", "¿Se animan a que hagamos una salita para vacunar?"). Este tipo de pseudopreguntas fue mucho menos frecuentes en la situación de lectura.

En cuanto a la comparación entre los tipos de preguntas en ambas situaciones, no se encontraron diferencias entre las preguntas cerradas literales versus inferenciales que las maestras realizaron en situaciones de juego y de lectura, $\chi^{2}(1, n=1614)=2,72, p=0,099$, mientras que realizaron más preguntas abiertas literales en situaciones de juego que en la lectura de cuentos, $\chi^{2}(1, n=238)=33,51, p<0,001$.

Respecto de las emisiones de los niños/as, se analizaron 2407 respuestas (69,5\% en juegos y 30,5\% en cuentos). En la Tabla 4 se presenta la distribución correspondiente a las situaciones de juego y cuento.

Tabla 4

Tipo y Pertinencia de Respuestas Efectuadas por los Niños/as

\begin{tabular}{lrrrr}
\hline & \multicolumn{2}{c}{ Juego } & \multicolumn{2}{c}{ Cuento } \\
\cline { 2 - 5 } Tipo y pertinencia de respuesta & Frecuencia & $\%$ & Frecuencia & $\%$ \\
\hline Literal relevante & 1369 & 81,8 & 615 & 83,9 \\
Literal irrelevante & 31 & 1,8 & 16 & 2,2 \\
Inferencial relevante & 268 & 16,0 & 98 & 13,4 \\
Inferencial irrelevante & 6 & 0,4 & 4 & 0,5 \\
Total & 1674 & 100,0 & 733 & 100,0 \\
\hline
\end{tabular}

En ambas situaciones las respuestas de los niños/as fueron pertinentes a la pregunta formulada por la maestra. En su mayoría estas respuestas fueron literales. La distribución de los tipos de respuesta de los niños/as en juego y cuento no fue distinta, $\chi^{2}(3, n=2407)=0,13, p=0,343$.

En cuanto a los seguimientos efectuados por las maestras, en la Tabla 5 se presenta la distribución de los tipos de seguimiento, tanto para juego como para cuento.

Hubo diferencias en los seguimientos de bajo y alto nivel entre juego y cuento, $\chi^{2}(7, n=884)=38,60$, $p<0,001$. Los de alto nivel se dieron mayormente en cuento, aunque en ambas situaciones los seguimientos fueron preponderantemente de bajo nivel. En cuanto a su distribución, el uso de la confirmación fue el más frecuente en ambas situaciones, mientras que los otros tipos de seguimientos tuvieron una frecuencia menor y se distribuyeron de manera distinta.

En relación a los seguimientos de alto nivel, el más frecuente fue el uso de elaboraciones, especialmente en situaciones de lectura, mientras que los restantes tipos de seguimientos se distribuyeron de manera distinta en cada situación.

\section{Análisis Secuencial}

\section{Tipo de Respuesta según Pregunta en Situaciones de Juego y Cuento}

En esta sección se presentan los resultados obtenidos en el cálculo de probabilidades transicionales entre el tipo de pregunta efectuada por la maestra y el tipo de respuesta de los niños/as, tanto para situaciones de juego como de lectura. En la Tabla 6 se observa que en ambas situaciones el tipo y complejidad de la pregunta realizada por la maestra determina, en la mayoría de los casos, la complejidad de la respuesta proporcionada por los niños/as. En efecto, el inicio de una interacción PRS con una pregunta cerrada literal tuvo una alta probabilidad de respuesta literal relevante. Asimismo, el inicio de la interacción con una pregunta cerrada inferencial tuvo asociada una mayor probabilidad de respuesta inferencial. En el caso de que la interacción comenzara con una pregunta abierta literal, se observó una alta probabilidad de una respuesta literal relevante en el juego, mientras que en situaciones de cuento se encontró una sola pregunta abierta literal. 
Tabla 5

Tipos de Seguimiento Efectuados por las Maestras

\begin{tabular}{|c|c|c|c|c|c|}
\hline \multirow{2}{*}{\multicolumn{2}{|c|}{ Tipo de seguimiento }} & \multicolumn{2}{|c|}{ Juego } & \multicolumn{2}{|c|}{ Cuento } \\
\hline & & Frecuencia & $\%$ & Frecuencia & $\%$ \\
\hline \multirow{5}{*}{ De bajo nivel } & Confirmación & 191 & 34,8 & 88 & 26,3 \\
\hline & Evaluación & 97 & 17,7 & 28 & 8,3 \\
\hline & Falsificación & 28 & 5,1 & 9 & 2,7 \\
\hline & Respuesta & 89 & 16,2 & 80 & 23,9 \\
\hline & Cierre & 5 & 0,9 & 5 & 1,5 \\
\hline \multirow{3}{*}{ De alto nivel } & Elaboración & 73 & 13,3 & 69 & 20,6 \\
\hline & Pista & 4 & 0,7 & 6 & 1,8 \\
\hline & Nueva pregunta & 62 & 11,3 & 50 & 14,9 \\
\hline Total & & 549 & 100,0 & 335 & 100,0 \\
\hline
\end{tabular}

Tabla 6

Probabilidades Transicionales entre Pregunta de la Maestra y Respuesta Infantil: Frecuencias y Porcentajes

\begin{tabular}{|c|c|c|c|c|c|c|c|c|c|c|}
\hline \multirow[b]{2}{*}{ Tipo de pregunta } & \multicolumn{5}{|c|}{ Juego } & \multicolumn{5}{|c|}{ Cuento } \\
\hline & $\begin{array}{c}\text { RLR } \\
N \\
(\%)\end{array}$ & $\begin{array}{c}\text { RLI } \\
N \\
(\%)\end{array}$ & $\begin{array}{c}\text { RIR } \\
N \\
(\%)\end{array}$ & $\begin{array}{c}\mathrm{RII} \\
N \\
(\%)\end{array}$ & Total & $\begin{array}{c}\text { RLR } \\
N \\
(\%)\end{array}$ & $\begin{array}{c}\text { RLI } \\
N \\
(\%)\end{array}$ & $\begin{array}{c}\text { RIR } \\
N \\
(\%)\end{array}$ & $\begin{array}{c}\text { RII } \\
N \\
(\%)\end{array}$ & Total \\
\hline Cerrada literal & $\begin{array}{c}795 \\
(96,6)\end{array}$ & $\begin{array}{c}15 \\
(1,8)\end{array}$ & $\begin{array}{c}9 \\
(1,1)\end{array}$ & $\begin{array}{c}4 \\
(0,5)\end{array}$ & 823 & $\begin{array}{c}391 \\
(95,6)\end{array}$ & $\begin{array}{c}9 \\
(2,2)\end{array}$ & $\begin{array}{c}9 \\
(2,2)\end{array}$ & 0 & 409 \\
\hline Cerrada inferencial & $\begin{array}{c}51 \\
(30,4)\end{array}$ & $\begin{array}{c}5 \\
(3,0)\end{array}$ & $\begin{array}{c}112 \\
(66,6)\end{array}$ & 0 & 168 & $\begin{array}{c}20 \\
(30,8)\end{array}$ & $\begin{array}{c}4 \\
(6,2)\end{array}$ & $\begin{array}{c}41 \\
(63,0)\end{array}$ & 0 & 65 \\
\hline Abierta literal & $\begin{array}{c}100 \\
(96,2)\end{array}$ & $\begin{array}{c}2 \\
(1,9)\end{array}$ & $\begin{array}{c}2 \\
(1,9)\end{array}$ & 0 & 104 & $\begin{array}{c}1 \\
(100,0)\end{array}$ & 0 & 0 & 0 & 1 \\
\hline Abierta inferencial & $\begin{array}{c}9 \\
(12,0)\end{array}$ & $\begin{array}{c}3 \\
(4,0)\end{array}$ & $\begin{array}{c}61 \\
(81,3)\end{array}$ & $\begin{array}{c}2 \\
(2,7)\end{array}$ & 75 & $\begin{array}{c}6 \\
(25,0)\end{array}$ & 0 & $\begin{array}{c}14 \\
(58,3)\end{array}$ & $\begin{array}{c}4 \\
(16,7)\end{array}$ & 24 \\
\hline Total & 955 & 25 & 184 & 6 & 1170 & 418 & 13 & 64 & 4 & 499 \\
\hline
\end{tabular}

Nota. Juego: $\chi^{2}(3, n=499)=741,43, p<0,01$. Cuento: $\chi^{2}(3, n=499)=324,63, p<0,01$. RLR. = Respuesta literal relevante; $\mathrm{RLI}=$ Respuesta literal irrelevante; RIR $=$ Respuesta inferencial relevante; RII = Respuesta inferencial irrelevante.

\section{Tipo de Seguimiento según Respuesta en Situaciones de Juego y Cuento}

Aquí se presentan los resultados obtenidos del cálculo de probabilidades transicionales entre el tipo de respuesta emitida por los niños/as (literal relevante, literal irrelevante, inferencial relevante, inferencial irrelevante) y el tipo de seguimiento (de bajo nivel o alto nivel) efectuado por la maestra en ambas situaciones. Para analizar los seguimientos se tomó en cuenta el realizado tanto inmediatamente después de una respuesta como dos turnos después, es decir, cuando un niño/a da una respuesta y luego otro (o él mismo) da 
otra respuesta antes de que la maestra haga un seguimiento. No se analizaron los seguimientos hechos después de tres turnos de respuesta. En la Tabla 7 se presentan los resultados obtenidos en el análisis.

\section{Tabla 7}

Probabilidades Transicionales entre Tipo de Respuesta Infantil y Seguimiento de la Maestra

\begin{tabular}{|c|c|c|c|c|c|c|}
\hline \multirow[b]{2}{*}{ Tipo de respuesta infantil } & \multicolumn{3}{|c|}{ Juego } & \multicolumn{3}{|c|}{ Cuento } \\
\hline & $\begin{array}{c}\mathrm{SBN} \\
N \\
(\%)\end{array}$ & $\begin{array}{c}\text { SAN } \\
N \\
(\%)\end{array}$ & Total & $\begin{array}{c}\text { SBN } \\
N \\
(\%)\end{array}$ & $\begin{array}{c}\text { SAN } \\
N \\
(\%)\end{array}$ & Total \\
\hline Respuesta literal relevante & $\begin{array}{c}333 \\
(77,3)\end{array}$ & $\begin{array}{c}98 \\
(22,7)\end{array}$ & 431 & $\begin{array}{c}144 \\
(65,2)\end{array}$ & $\begin{array}{c}77 \\
(34,8)\end{array}$ & 221 \\
\hline Respuesta literal irrelevante & $\begin{array}{c}7 \\
(100,0)\end{array}$ & 0 & 7 & $\begin{array}{c}5 \\
(62,5)\end{array}$ & $\begin{array}{c}3 \\
(37,5)\end{array}$ & 8 \\
\hline Respuesta inferencial relevante & $\begin{array}{c}35 \\
(59,3)\end{array}$ & $\begin{array}{c}24 \\
(40,7)\end{array}$ & 59 & $\begin{array}{c}24 \\
(57,1)\end{array}$ & $\begin{array}{c}18 \\
(42,9)\end{array}$ & 42 \\
\hline Respuesta inferencial irrelevante & $\begin{array}{c}1 \\
(50,0)\end{array}$ & $\begin{array}{c}1 \\
(50,0)\end{array}$ & 2 & $\begin{array}{c}1 \\
(100,0)\end{array}$ & 0 & 1 \\
\hline Total & 376 & 123 & 499 & 174 & 98 & 272 \\
\hline
\end{tabular}

Nota. Juego: $\chi^{2}(3, n=499)=11,99, p=0,01$. Cuento: $\chi^{2}(3, n=272)=1,56, p=0,67$. SBN = Seguimiento de bajo nivel; $\mathrm{SAN}=$ Seguimiento de alto nivel.

Los resultados muestran que, frente a cualquier respuesta, la mayoría de los seguimientos fue de bajo nivel. Sin embargo, esta probabilidad varió según el tipo de respuesta proporcionada. Así, después de que los niños/as dieran respuestas literales relevantes, hubo una alta probabilidad de encontrar seguimientos de bajo nivel, tanto en situaciones de juego como de lectura de cuento, mientras que después de respuestas inferenciales relevantes, la probabilidad de encontrar seguimientos de bajo nivel disminuyó en situaciones de juego.

En cuanto a los seguimientos de alto nivel, hubo una mayor probabilidad de que las maestras los emplearan después de que los niños/as dieran respuestas inferenciales relevantes, en situaciones de juego, siendo menos probables después de respuestas literales relevantes.

\section{Probabilidades Transicionales entre Pregunta y Seguimiento de la Maestra}

En cuanto al cálculo de probabilidades transicionales entre el tipo de pregunta y el nivel de seguimiento efectuado por las maestras, se encontró que tanto en juego como en cuento no fue significativo, lo que significa que sin importar el tipo de pregunta de la maestra (abierta o cerrada), la probabilidad de que el seguimiento fuera de bajo nivel es alta en juego y mediana en cuento (ver Tabla 8).

\section{Discusión}

El principal resultado de esta investigación muestra que, tanto en situaciones de juego de rincón como de lectura de cuento, el tipo y complejidad de la pregunta con que la maestra inició la interacción determinó fuertemente la complejidad de la respuesta de los niños/as, mientras que, en ambas situaciones, los seguimientos de las maestras frente a las respuestas de los niños/as fueron mayormente de bajo nivel, independientemente del tipo de pregunta utilizado. La complejidad de la respuesta de los niños/as también influyó en la calidad de los seguimientos, pero solo en situaciones de juego de rincón. Se encontró que la mayoría de las preguntas efectuadas por las maestras fueron literales y cerradas. En consecuencia, las respuestas infantiles también fueron literales y los seguimientos proporcionados por la maestra, de bajo nivel. 
Estas características de las interacciones PRS fueron similares a las observadas en aulas preescolares de otros países (Dickinson \& Porche, 2011; Hiebert et al., 2003; Mascareño et al., 2016, 2017).

Tabla 8

Probabilidades Transicionales entre Tipo de Pregunta y Seguimiento Efectuado por las Maestras

\begin{tabular}{|c|c|c|c|c|c|c|}
\hline \multirow[b]{2}{*}{ Tipo de pregunta } & \multicolumn{3}{|c|}{ Juego } & \multicolumn{3}{|c|}{ Cuento } \\
\hline & $\begin{array}{c}\text { Seg. de } \\
\text { bajo nivel } \\
N \\
(\%)\end{array}$ & $\begin{array}{c}\text { Seg. de alto } \\
\text { nivel } \\
N \\
(\%)\end{array}$ & Total & $\begin{array}{c}\text { Seg. de } \\
\text { bajo nivel } \\
N \\
(\%)\end{array}$ & $\begin{array}{c}\text { Seg. de alto } \\
\text { nivel } \\
N \\
(\%)\end{array}$ & Total \\
\hline Pregunta cerrada literal & $\begin{array}{c}221 \\
(76,2)\end{array}$ & $\begin{array}{c}69 \\
(23,8)\end{array}$ & 290 & $\begin{array}{c}97 \\
(65,5)\end{array}$ & $\begin{array}{c}51 \\
(34,5)\end{array}$ & 148 \\
\hline Pregunta cerrada inferencial & $\begin{array}{c}22 \\
(71,0)\end{array}$ & $\begin{array}{c}9 \\
(29,0)\end{array}$ & 31 & $\begin{array}{c}10 \\
(50,0)\end{array}$ & $\begin{array}{c}10 \\
(50,0)\end{array}$ & 20 \\
\hline Pregunta abierta literal & $\begin{array}{c}20 \\
(83,3)\end{array}$ & $\begin{array}{c}4 \\
(16,6)\end{array}$ & 24 & 0 & 0 & 0 \\
\hline Pregunta abierta inferencial & $\begin{array}{c}17 \\
(74,0)\end{array}$ & $\begin{array}{c}6 \\
(26,0)\end{array}$ & 23 & $\begin{array}{c}7 \\
(50,0)\end{array}$ & $\begin{array}{c}7 \\
(50,0)\end{array}$ & 14 \\
\hline Total & 280 & 88 & 368 & 114 & 68 & 182 \\
\hline
\end{tabular}

Nota. Juego: $\chi^{2}(3, n=368)=1,20, p=0,76$; Cuento: $\chi^{2}(3, n=182)=2,85, p=0,42$.

Entre los resultados se destaca también que en ambas situaciones las maestras utilizaron más turnos de habla que los niños/as. No obstante, esta diferencia no impidió la participación infantil. De las emisiones producidas por las maestras, la gran mayoría fueron preguntas, lo cual evidencia que el foco de las secuencias estaba orientado a indagar información y, por tanto, la participación de los niños/as estaba orientada a proporcionar respuestas.

También se observó que las maestras realizaban preguntas en una proporción mayor en situaciones de juego que de lectura. Esto tuvo como consecuencia que la cantidad de respuestas infantiles también fuera mayor en juego y que la maestra efectuara más seguimientos. Por ende, la interacción dialógica entre maestras y niños/as fue más activa y sus intervenciones fueron en proporciones similares en situaciones de juegos de rincón. Estos hallazgos son consistentes con lo planteado tanto por Manrique y Rosemberg (2009), como por estudios que evidenciaron una correlación directa entre las preguntas de la maestra y la participación de los niños/as (Gest et al., 2006; Meacham et al., 2014).

La mayoría de las preguntas efectuadas por las maestras en ambas situaciones fue de bajo nivel, es decir, cerradas y literales, lo que concuerda con los hallazgos en aulas preescolares chilenas (González et al., 2008; Tornero et al., 2015). Además, esta clase de preguntas fue más frecuente en situaciones de lectura que de juego de rincón. Si bien el estudio de Tornero et al. (2015) encontró que en situaciones de lectura de cuentos las maestras realizaban más preguntas abiertas, un análisis posterior indicó que estas preguntas eran de baja complejidad, ya que se limitaban a recuperar o identificar información recientemente leída del texto y no promovían una elaboración por parte de los niños/as.

La aparición de un mayor número de preguntas cerradas literales en situaciones de lectura podría deberse a que, durante la lectura del texto, hay mucha información disponible en este. Las maestras, al manejar más conocimientos específicos que los niños/as, probablemente realizan preguntas muy restrictivas destinadas a evaluar si las respuestas de los niños/as son correctas o no, situación que concuerda con lo planteado por Smart y Marshall (2013), mientras que la aparición de menos preguntas cerradas literales y la presencia de preguntas abiertas literales en situaciones de juego podría explicarse porque durante el juego la información sobre lo que los niños/as realizan no es tan accesible para la maestra, por tanto, efectúa 
preguntas orientadas a participar en el juego y el diálogo (Girolametto et al., 2000; Girolametto \& Weitzman 2002; Kontos, 1999).

En lo que respecta a los seguimientos, fueron más frecuentes los de bajo nivel (confirmación, evaluación, falsificación, respuesta y cierre) en ambas situaciones. Estos hallazgos concuerdan con los de Dickinson et al. (2003) y Mascareño et al. (2016, 2017).

En cuanto a la distribución de los seguimientos de bajo nivel, la confirmación fue la más recurrente en juego y cuento, mientras que la respuesta fue más frecuente en situaciones de lectura que en juego. Estos resultados podrían explicarse porque la maestra, al conocer la información proporcionada por los textos narrativos, podía dar más respuestas consideradas correctas después de la contribución de los niños/as. Durante las situaciones de juegos de rincón, en cambio, fueron más frecuentes las confirmaciones y evaluaciones, ya que permiten promover y apoyar la actividad lúdica de los niños/as (Kontos, 1999).

Respecto de los seguimientos de alto nivel, considerados importantes porque facilitan el aprendizaje (Carlsen, 1991; Chin, 2006; Wang \& Wang, 2013), en general aparecieron con menor frecuencia, pero en situaciones de lectura fueron más utilizados que en juego. Dentro de este tipo de seguimientos, las elaboraciones fueron más frecuentes, seguidas de una nueva pregunta, mientras que la provisión de pistas casi no se observó. Posiblemente, durante la lectura la maestra, al conocer la información que proporcionan los textos, podía anticipar las posibles respuestas y estar preparada para expandir o reelaborar esa información. Durante el juego, en cambio, la maestra estaría atenta a las intervenciones infantiles, porque se trataba de información que los niños/as proporcionaban espontáneamente. Esta situación no la habilitaría, o lo haría en menor medida, a anticiparse a posibles respuestas de los niños/as.

La diferencia entre el nivel de seguimiento permite pensar que las situaciones de juego de rincón tienen una función diferente a la de lectura de cuentos. En el juego, la distribución de turnos fue más equilibrada: tanto las preguntas como los seguimientos estaban orientados a promover la participación y el desarrollo de la actividad. Estas observaciones coinciden con lo reportado por Manrique y Rosemberg (2009) y por Kontos (1999). En la lectura, en cambio, las preguntas y seguimientos tuvieron como objetivo potenciar la comprensión que tienen los niños/as de lo que se está leyendo e indagar si los niños/as recordaban información relevante.

En el presente estudio cobró especial relevancia el análisis de la secuencia PRS. En ambas situaciones el tipo y complejidad de la pregunta efectuada por la maestra determinó fuertemente la complejidad de la respuesta proporcionada por los niños/as, lo que concuerda con lo planteado por Mascareño et al. (2017).

Cabe destacar que al no contar con estudios que hayan analizado la secuencia PRS en situaciones de juego, ni con estudios comparando juego y cuento, el presente estudio constituye un aporte novedoso. Su originalidad radica en la comparación entre situaciones de juego y cuento en lo referido a la frecuencia de las preguntas, respuestas y seguimientos, así como en la relación entre las intervenciones del adulto y del niño/a en cada situación. Los resultados indican que la relación entre el habla de la maestra y de los niños/as es similar en ambas situaciones, al menos en cuanto a las secuencias más típicas de pregunta-respuestaseguimiento. Una diferencia interesante fue que la probabilidad de que la maestra proporcionara seguimientos después de las respuestas de los niños fue más baja en situaciones de juego. Esto sugiere que la situación de juego, al ser más ambigua y reducir la asimetría de conocimientos entre adulto y niño/a, podría alterar el balance de control sobre la situación, explicando la mayor participación verbal de los niños/as durante el juego. Este estudio muestra que en un contexto donde el juego de rincón forma parte de la rutina cotidiana de las aulas, puede funcionar como promotor del lenguaje, dada la alta participación de los niños/as en la actividad.

Los resultados en su conjunto conducen a reflexionar sobre las posibles implicancias didácticas del presente estudio. Resulta relevante proponer que en programas, tanto de formación docente inicial como continua, se destinaran espacios específicos para la puesta en práctica de preguntas más complejas o inferenciales abiertas por parte de las maestras. Estas preguntas permitirían que los niños/as proporcionen respuestas más extensas que, a su vez, posibiliten seguimientos de mayor nivel. También sería importante que se conciba el juego de rincón como una oportunidad, no solo para trabajar el desarrollo de habilidades sociales y emocionales, sino también para el aprendizaje del lenguaje, sin restringir su enseñanza a la lectura de cuentos. Asimismo, se podría potenciar un mayor desarrollo de habilidades lingüísticas en cualquier situación del aula, como ronda y merienda, entre otras, estimulando la generación la producción de respuestas complejas y sofisticadas. 
Una limitación de esta investigación se relaciona con la muestra. Las clases grabadas en audio corresponden a las efectuadas por maestras que trabajaban en establecimientos enmarcados en un contexto de vulnerabilidad social y que participaban en un programa de desarrollo profesional docente. Esto implica que las observaciones efectuadas no necesariamente serían representativas de lo que sucede cotidianamente en otras aulas de nivel inicial. Además, las maestras sabían que sus clases estaban siendo grabadas, lo que podría haber influido en su forma de realizar las actividades. No obstante, esta limitación también puede considerarse como una ventaja, puesto que las observaciones podrían dar cuenta de lo que las maestras conciben como una buena práctica. Como otros estudios en contextos latinoamericanos (Medina Morales et al., 2015; Mendive et al., 2016), las maestras sabían a priori el día que sería grabada su clase. Esto permitiría inferir, en concordancia con Medina Morales et al. (2015), que la situación de grabación pudo haber influido en su quehacer pedagógico y las maestras se limitarían a realizar preguntas que saben que los niños/as van a responder, sin plantear desafíos mayores. Así, los resultados de este estudio pueden generar sugerencias específicas para el trabajo en el aula, como, por ejemplo, modelar preguntas inferenciales y abiertas de forma explícita para favorecer el lenguaje en los niños/as.

Cabe destacar que, aunque se haya intervenido, de todos modos se encontró que las maestras realizaron preguntas y seguimientos de bajo nivel y, por tanto, las respuestas de los niños/as fueron de bajo nivel. En futuras investigaciones podría realizarse un estudio similar, pero comparando aulas de nivel inicial de diferentes NSE. Asimismo, resultaría interesante comparar los resultados aquí encontrados con interacciones de niños/as y maestras que no se encuentren en el marco de un programa de intervención, para evaluar posibles diferencias debidas a la capacitación recibida. Por último, también se podrían incluir observaciones de otras situaciones de la jornada escolar, además de lectura de cuentos y juego de rincón, tales como el momento del saludo inicial o ronda, puesto que tiende a ser un espacio de interacción dialogante entre docentes y niños/as y quizás podrían identificarse secuencias de pregunta, respuesta y seguimiento distintas a los resultados de este estudio.

\section{Referencias}

Beck, I. L., McKeown, M. G. \& Kucan, L. (2013). Bringing words to life: Robust vocabulary instruction (2a ed.). Guilford Press.

Bruckner, C. T., Yoder, P. \& MacLean Jr., W. E. (2006). Interpreting Kappa in observational research: Baserate matters. American Journal on Mental Retardation, 111(6), 433-441. https://doi.org/10.1352/0895-8017(2006)111[433:IKIORB]2.0.CO;2

Bruner, J. (1986). El habla del niño. Paidós.

Cain, K. \& Oakhill, J. V. (1999). Inference making ability and its relation to comprehension failure in young children. Reading and Writing, 11(5-6), 489-503. https://doi.org/10.1023/A:1008084120205

Cain, K., Oakhill, J. \& Bryant, P. (2004). Children's reading comprehension ability: Concurrent prediction by working memory, verbal ability, and component skills. Journal of Educational Psychology, 96(1), 31-42. https://doi.org/10.1037/0022-0663.96.1.31

Carlsen, W. S. (1991). Questioning in classrooms: A sociolinguistic perspective. Review of Educational Research, 61(2), 157-178. https://doi.org/10.3102/00346543061002157

Chafy, M. E. \& Elkhouzai, E. (2014). Classroom interaction: Investigating the forms and functions of teacher questions in Moroccan primary school. International Journal of Innovation and Applied Studies, 6(3), 352-361. https://search.proquest.com/openview/88e4dba4c83cd0775d5cea21ad877bb3/1?pq-origsite=gscholar\&cbl=2031961

Chin, C. (2006). Classroom interaction in science: Teacher questioning and feedback to students' responses. International Journal of Science Education, 28(11), 1315-1346. https://doi.org/10.1080/09500690600621100

Dickinson, D. K., McCabe, A., Anastasopoulos, L., Peisner-Feinberg, E. S. \& Poe, M. D. (2003). The comprehensive language approach to early literacy: The interrelationships among vocabulary, phonological sensitivity, and print knowledge among preschool-aged children. Journal of Educational Psychology, 95(3), 465-481. https://doi.org/10.1037/0022-0663.95.3.465

Dickinson, D. K. \& Porche, M. V. (2011). Relation between language experiences in preschool classrooms and children's kindergarten and fourth-grade language and reading abilities. Child Development, 82(3), 870-886. https://doi.org/10.1111/j.1467-8624.2011.01576.x

Dickinson, D. K. \& Smith, M. W. (1994). Long-term effects of preschool teachers' book readings on low-income children's vocabulary and story comprehension. Reading Research Quarterly, 29(2), 104-122. https://doi.org/10.2307/747807

García Cernaz, S. (2019). Un estudio longitudinal-microanalítico del desarrollo del juego simbólico infantil a partir de técnicas de análisis de las artes temporales. Anuario Temas en Psicología, 5(1), 160-167. https://revistas.unlp.edu.ar/AnuarioPsicologia/article/view/9781/8581

Gest, S. D., Holland-Coviello, R., Welsh, J. A., Eicher-Catt, D. L. \& Gill, S. (2006). Language development subcontexts in head start classrooms: Distinctive patterns of teacher talk during free play, mealtime, and book reading. Early Education and Development, 17(2), 293-315. https://doi.org/10.1207/s15566935eed1702_5

Girolametto, L. \& Weitzman, E. (2002). Responsiveness of child care providers in interactions with toddlers and preschoolers. Language, Speech and Hearing Services in Schools, 33(4), 268-281. https://doi.org/10.1044/0161-1461(2002/022)

Girolametto, L., Weitzman, E., van Lieshout, R. \& Duff, D. (2000). Directiveness in teachers' language input to toddlers and preschoolers in day care. Journal of Speech, Language, and Hearing Research, 43(5), 1101-1114. https://doi.org/10.1044/jslhr.4305.1101

González, J., Preiss, D. \& San Martín, E. (2008). Evaluando el discurso docente: desarrollo de un modelo de Rasch a partir de la evidencia audiovisual de profesores chilenos de primer ciclo de educación básica en el área de lenguaje. Revista Iberoamericana de Evaluación Educativa, 1(2), 137-147. https://dialnet.unirioja.es/servlet/articulo?codigo=2789100 
Hiebert, J., Gallimore, R., Garnier, H., Givvin, K. B., Hollingsworth, H., Jacobs, J., Chui, A. M. -Y., Smith, M., Kersting, N., Manaster, A., Tseng, E., Etterbeek, W., Manaster, C., Gonzales, P. \& Stigler, J. (2003). Teaching mathematics in seven countries: Results from the TIMSS 1999 video study. U.S. Department of Education, National Center for Education Statistics. https://nces.ed.gov/pubs2003/2003013.pdf

Ibañez, M. I., Ramírez, M. L. \& Rosemberg, C. R. (2018). "Salga de acá, vaya para allá": las características léxicas y pragmáticas del discurso docente en el jardín maternal. Revista de Psicología de la Pontificia Universidad Católica Argentina, 14(27), 111-123. https://erevistas.uca.edu.ar/index.php/RPSI/article/view/1362/1287

Kontos, S. (1999). Preschool teachers' talk, roles, and activity settings during free play. Early Childhood Research Quarterly, 14(3), 363382. https://doi.org/10.1016/S0885-2006(99)00016-2

Lineamientos curriculares para la educación inicial, Gobierno de la Provincia de Entre Ríos, Consejo General de Educación (2008). http://www.bnm.me.gov.ar/giga1/documentos/EL006381.pdf

Lineamientos para el comportamiento ético en las ciencias sociales y humanidades (CSyH), Resolución 2857/06, Ministerio de Educación, Ciencia y Tecnología, Secretaría de Ciencia, Tecnología e Innovación Productiva, Consejo Nacional de Investigaciones Científicas y Técnicas (2006). https://www.conicet.gov.ar/wp-content/uploads/RD-20061211-2857.pdf

Manrique, M. S. \& Rosemberg, C. R. (2009). El lenguaje infantil en situaciones de juego en el jardín de infantes. Summa Psicológica UST, $6(2), 105-118$. https://doi.org/10.18774/448x.2009.6.66

Mascareño, M., Deunk, M. I., Snow, C. E. \& Bosker, R. J. (2017). Read-alouds in kindergarten classroom: A moment-by-moment approach to analyzing teacher-child interactions. European Early Childhood Education Research Journal, 25(1), 136-152. https://doi.org/10.1080/1350293X.2016.1266226

Mascareño, M., Snow, C. E., Deunk, M. I. \& Bosker, R. J. (2016). Language complexity during read-alouds and kindergartners' vocabulary and symbolic understanding. Journal of Applied Developmental Psychology, 44, 39-51. https://doi.org/10.1016/j.appdev.2016.02.001

Marradi, A., Archenti, N. \& Piovani, J. I. (2007). Metodología de las ciencias sociales. Emecé.

Meacham, S., Vukelich, C., Han, M. \& Buell, M. (2014). Preschool teachers' questioning in sociodramatic play. Early Childhood Research Quarterly, 29(4), 562-573. https://doi.org/10.1016/j.ecresq.2014.07.001

Medina Morales, L., Valdivia Barrios, A., Gaete Moscoso, R. \& Galdames Franco, V. (2015). ¿Cómo enseñan a leer los profesores de $1^{\circ}$ y $2^{\circ}$ básico en un contexto de evaluación de desempeño docente en Chile? Estudios Pedagógicos (Valdivia), 41(1), 183-198. https://doi.org/10.4067/S0718$\underline{07052015000100011}$

Mehan, H. (1979). Learning lessons: Social organization in the classroom. Harvard University Press.

Mendive, S., Weiland, C., Yoshikawa, H. \& Snow, C. (2016). Opening the black box: Intervention fidelity in a randomized trial of a preschool teacher professional development program. Journal of Educational Psychology, 108(1), 130-145. https://doi.org/10.1037/edu0000047

Migdalek, M. J. \& Rosemberg, C. R. (2014). Juegos con reglas convencionales: un análisis multimodal de la regulación de la situación lúdica por parte de la maestra. Educación, Lenguaje y Sociedad, 11, 1-25. https://doi.org/10.19137/els-2014-111108

Ortiz, C. \& Lissi, M. R. (2019). ¿Cómo enseñan a comprender textos escritos los profesores de segundo ciclo de educación básica? Caracterización a partir de la observación de clases [Manuscrito no publicado]. Escuela de Psicología, Pontificia Universidad Católica de Chile.

Preiss, D. D. (2009). The Chilean instructional pattern for the teaching of language: A video-survey study based on a national program for the assessment of teaching. Learning and Individual Differences, 19(1), 1-11. https://doi.org/10.1016/j.lindif.2008.08.004

Ramírez-Esparza, N., García-Sierra, A. \& Kuhl, P. K. (2014). Look who's talking: Speech style and social context in language input to infants are linked to concurrent and future speech development. Developmental Science, 17(6), 880-891. https://doi.org/10.1111/desc.12172

Reese, E., Cox, A., Harte, D. \& McAnally, H. (2003). Diversity in adults' styles of reading books to children. En A. van Kleeck, S. A. Stahl \& E. B. Bauer (Eds.), On reading books to children: Parents and teachers (pp. 37-57). Lawrence Erlbaum.

Rosemberg, C. R. (24-28 de septiembre de 2000). Los mecanismos de influencia educativa en los primeros años de la escolaridad. Contextos de aprendizaje y "contextos cognitivos" [Presentación de ponencia]. 23a Reunião Anual da Associação Nacional de PósGraduação e Pesquisa em Educação, Caxambu, Minas Gerais, Brasil.

Rosemberg, C. R. (2008). El lenguaje y el juego en la educación infantil. En P. Sarlé (Coord.), Enseñar en clave de juego: enlazando juegos $y$ contenidos (pp. 79-99). Novedades Educativas.

Rosemberg, C. R. \& Borzone, A. M. (2010). Programa de Promoción del Desarrollo Lingüístico y Cognitivo para los Jardines de Infantes de la Provincia de Entre Ríos. Fundación Arcor/Consejo Nacional de Investigaciones Científicas y Técnicas. https://www.academia.edu/1964758/PROGRAMA DE PROMOCI\%C3\%93N DEL DESARROLLO LING\%C3\%9C\%C3\%8DSTICO _Y_COGNITIVO_PARA_LOS_JARDINES_DE_INFANTES_DE_LA_PROVINCIA_DE_ENTRE_R\%C3\%8DOS

Rosemberg, C. R., Silva, M. L. \& Borzone, A. M. (2010). Programa de Promoción del Desarrollo Linguístico y Cognitivo para los Jardines de Infantes de la Provincia de Entre Ríos. Módulo 1: El desarrollo del lenguaje y el desarrollo cognitivo de niños en el jardín de infantes. Fundación Arcor/Consejo Nacional de Investigaciones Científicas y Técnicas. https://www.academia.edu/1964758/PROGRAMA DE PROMOCI\%C3\%93N DEL DESARROLLO LING\%C3\%9C\%C3\%8DSTIC O_Y_COGNITIVO_PARA_LOS_JARDINES_DE_INFANTES_DE_LA_PROVINCIA_DE_ENTRE_R\%C3\%8DOS

Sarlé, P. (2000). El juego dramático, la educación infantil y el aprendizaje escolar. Psykhe, 9(2), 41-53. http://psykhe.cl/index.php/psykhe/article/view/448 Sarlé, P. M. (2006). Enseñar el juego y jugar la enseñanza. Paidós.

Sembiante, S. F., Dynia, J. M., Kaderavek, J. N. \& Justice, L. M. (2018). Teachers' literal and inferential talk in early childhood and special education classrooms. Early Education and Development, 29(1), 14-30. https://doi.org/10.1080/10409289.2017.1362916

Sinclair, J. M. \& Coulthard, R. M. (1975). Towards an analysis of discourse: The English used by teachers and pupils. Oxford University Press.

Smart, J. B. \& Marshall, J. C. (2013). Interactions between classroom discourse, teacher questioning, and student cognitive engagement in middle school science. Journal of Science Teacher Education, 24(2), 249-267. https://doi.org/10.1007/s10972-012-9297-9

Strasser, K., Darricades, M., Mendive, S. \& Barra, G. (2018). Instructional activities and the quality of language in Chilean preschool classrooms. Early Education and Development, 29(3), 357-378. https://doi.org/10.1080/10409289.2018.1429765

Tornero, B., Ramaciotti, A., Truffello, A. \& Valenzuela, F. (2015). Nivel cognitivo de las preguntas que formulan las educadoras de párvulos. Educación y Educadores, 18(2), 261-283. https://doi.org/10.5294/edu.2015.18.2.5

Treviño, E., Varela, C., Romo, F. \& Nuñez, V. (2015). Presencia de lenguaje académico en las educadoras de párvulos y su relación con el desarrollo del lenguaje de los niños. Calidad en la Educación, 43, 137-168. https://doi.org/10.4067/S0718-45652015000200005

van Kleeck, A., Vander Woude, J. \& Hammett, L. (2006). Fostering literal and inferential language skills in head start preschoolers with language impairment using scripted book-sharing discussions. American Journal of Speech-Language Pathology, 15(1), 85-95. https://doi.org/10.1044/1058-0360(2006/009) 
Wang, K. \& Wang, X. (2013). Promoting knowledge construction and cognitive development: A case study of teacher's questioning. Theory and Practice in Language Studies, 3(8), 1387-1392. https://doi.org/10.4304/tpls.3.8.1387-1392

Wasik, B. A. \& Bond, M. A. (2001). Beyond the pages of a book: Interactive book reading and language development in preschool classrooms. Journal of Educational Psychology, 93(2), 243-250. https://doi.org/10.1037/0022-0663.93.2.243

Zimmerman, F. J., Gilkerson, J., Richards, J. A., Christakis, D. A., Xu, D., Gray, S. \& Yapanel, U. (2009). Teaching by listening. The importance of adult-child conversations to language development. Pediatrics, 124(1), 342-349. https://doi.org/10.1542/peds.2008-2267

Fecha de recepción: Febrero de 2019.

Fecha de aceptación: Mayo de 2020. 